\title{
Dynamics of cellular automata in non-compact spaces
}

\author{
Enrico Formenti \\ Université de Nice Sophia Antipolis, \\ Laboratoire I3S - UNSA/CNRS UMR 6070 \\ 2000 route des Lucioles BP 12106903 Sophia Antipolis, France \\ Petr Kůrka \\ Center for Theoretical Study, \\ Academy of Sciences and Charles University in Prague, \\ Jilská 1, CZ-11000 Praha 1, Czechia
}

\section{Contents}

1 Glossary 2

2 Definition 2

3 Introduction 3

4 Dynamical systems 4

5 Cellular automata 5

6 Submeasures 5

7 The Cantor space 6

8 The periodic space $\quad 6$

$\begin{array}{lll}9 & \text { The Toeplitz space } & 7\end{array}$

10 The Besicovitch space 10

11 The generic space 11

12 The space of measures $\quad 12$

13 The Weyl space 13

14 Examples $\quad 14$

15 Future directions $\quad 15$

16 Primary literature $\quad 16$

$\begin{array}{ll}17 \text { Books and reviews } & 17\end{array}$ 


\section{Glossary}

Almost equicontinuous CA: a $\mathrm{CA}$ which has at least one equicontinuous configuration.

Attraction basin: the set of configurations whose orbit is eventually attracted by an attractor.

Attractor: a closed invariant set which attracts all orbits in some of its neighbourhood.

Besicovitch pseudometrics: a pseudo-metric that quantifies the upper-density of differences.

Blocking word: a word that interrupts the information flow. A configuration containing an infinite number of blocking words both to the right and to the left gives rise to an equicontinuous configuration.

Equicontinuous CA: a CA in which all configurations are equicontinuous.

Equicontinuous configuration: a configuration for which nearby configurations remain close.

Expansive CA: two distinct configurations, no matter how close, eventually separate during the evolution.

Generic space: the space of configurations for which upper-density and lowerdensity coincide.

Sensitive CA: in any neighbourhood of any configuration there exists a configuration such that the orbits of the two configurations eventually separate.

Spreading set: a clopen invariant set propagating both to the left and to the right.

Toeplitz space: the space of regular quasi-periodic configurations.

Weyl pseudometrics: a pseudo-metric that quantifies the upper density of differences with respect to all possible cell indices.

\section{Definition}

In topological dynamics, the assumption of compactness is usually adopted as it has far reaching consequences. Each compact dynamical system has an almost periodic point, contains a minimal subsystem, and each trajectory has a limit point. Nevertheless, there are important examples of non-compact dynamical systems like linear systems on $\mathbb{R}^{n}$ and the theory should cover these examples as well. The study of dynamics of cellular automata (CA) in the compact Cantor space of symbolic sequences starts with Hedlund [7] and is by now a firmly established discipline (see e.g., Kurka [15]). The study of dynamics of CA in non-compact spaces like Besicovitch or Weyl spaces is more recent and provides an interesting alternative perspective.

The study of dynamics of cellular automata in non-compact spaces has at least two distinct origins. The first concerns the study of dynamical properties on peculiar countable dense sub-spaces of the Cantor space (the space of finite configuration or the space of spatially periodic configurations, for instance). The idea is that on those spaces, some properties are easier to prove than on the full Cantor space. Once a property is proved on such a sub-space, one can try to lift it to the original Cantor space by using denseness. Another advantage is that the configurations on these spaces are easily representable 
on computers. Indeed, computer simulations and practical applications of CA usually take place in these subspaces.

The second origin is connected to the question of suitability of the classical Cantor topology for the study of chaotic behavior of CA and of symbolic systems in general. We briefly recall the motivations. Consider sensitivity to initial conditions for a CA in the Cantor topology. The shift map $\sigma$, which is a very simple CA, is sensitive to initial conditions since small perturbations far from the central region are eventually brought to the central part. However, from an algorithmic point of view, the shift map is very simple. We are inclined to regard a system as chaotic if its behavior cannot easily be reconstructed. This is not the case of the shift map whose chaoticity is more an artifact of the Cantor metric, rather than an intrinsic property of the system. Therefore, one may want to define another metric in which sensitive CA not only transport information (like the shift map) but also build/destroy new information at each time step.

This basic requirement stimulated the quest for alternative topologies to the classical Cantor space. This lead first to the Besicovitch topology and then to the Weyl topology in Cattaneo et al [4]. used to investigate almost periodic real functions (see Besicovitch [1] or Iwanik [9]). Both these pseudometrics can be defined starting from suitable semi-measures on the set $\mathbb{Z}$ of integers. This way of construction had a Pandora effect opening the way to many new interesting topological spaces. Some of them are reported in this paper; others can be found in Cervelle and Formenti [5].

Each topology focuses on some peculiar aspects of the dynamics under study but all of them have a common denominator, namely non-compactness.

\section{Introduction}

A given $\mathrm{CA}$ in alphabet $A$ can be regarded as a dynamical system in several topological spaces: Cantor configuration space $\mathcal{C}_{A}$, space $\mathcal{M}_{A}$ of shift-invariant Borel probability measures on $A^{\mathbb{Z}}$, the Weyl space $\mathcal{W}_{A}$, the Besicovitch space $\mathcal{B}_{A}$, the generic space $\mathcal{G}_{A}$, the Toeplitz space $\mathcal{T}_{A}$ and the periodic space $\mathcal{P}_{A}$. We refer to various topological properties of these systems by prefixing the name of space in question. Basic results correlate various dynamical properties of CA in these spaces.

The Cantor topology corresponds to the point of view of an observer who can distinguish only a finite central part of a configuration and sites outside this central part of the configuration are not taken into account. The Besicovitch and Weyl topologies, on the other hand, correspond to a god-like position of someone who sees whole configurations and can distinguish the frequency of differences. In the Besicovitch topology, the centers of configurations still play a distinguished role, as the frequencies of differences are computed from the center. In the Weyl topology, on the other hand, no site has a privileged position. Both Besicovitch and Weyl topologies are defined by pseudometrics. Different configurations can have zero distance and the topological space consists of equivalence classes of configurations which have zero distance.

The generic space $\mathcal{G}_{A}$ is a subspace of the Besicovich space of those configurations, in which each finite word has a well defined frequency. These frequencies define a Borel probability measure on the Cantor space of configurations, so we have a projection from the generic space $\mathcal{G}_{A}$ to the space $\mathcal{M}_{A}$ of Borel probability measures equipped with the weak* topology. This is a natural space for investigating the dynamics of CA on random configurations.

The Toeplitz space $\mathcal{T}_{A}$ consists of regular quasi-periodic configurations. This means that each pattern repeats periodically but different patterns have different periods. The Besicovitch and Weyl pseudometrics are actually metrics on the Toeplitz space and moreover they coincide on $\mathcal{T}_{A}$. 


\section{Dynamical systems}

A dynamical system is a continuous map $F: X \rightarrow X$ of a nonempty metric space $X$ to itself. The $n$-th iteration $F^{n}: X \rightarrow X$ of $F$ is defined by $F^{0}(x)=x$, $F^{n+1}(x)=F\left(F^{n}(x)\right)$. A point $x \in X$ is fixed, if $F(x)=x$. It is periodic, if $F^{n}(x)=x$ for some $n>0$. The least positive $n$ with this property is called the period of $x$. The orbit of $x$ is the set $\mathcal{O}(x)=\left\{F^{n}(x): n>0\right\}$. A set $Y \subseteq X$ is positively invariant, if $F(Y) \subseteq Y$ and strongly invariant if $F(Y)=Y$. A point $x \in X$ is equicontinuous $\left(x \in \mathcal{E}_{F}\right)$ if the family of maps $F^{n}$ is equicontinuous at $X$, i.e. $x \in \mathcal{E}_{F}$ iff

$$
(\forall \varepsilon>0)(\exists \delta>0)\left(\forall y \in B_{\delta}(x)\right)(\forall n>0)\left(d\left(F^{n}(y), F^{n}(x)\right)<\varepsilon\right) .
$$

The system $(X, F)$ is almost equicontinuous if $\boldsymbol{E}_{F} \neq \emptyset$ and equicontinuous, if

$$
(\forall \varepsilon>0)(\exists \delta>0)(\forall x \in X)\left(\forall y \in B_{\delta}(x)\right)(\forall n>0)\left(d\left(F^{n}(y), F^{n}(x)\right)<\varepsilon\right) .
$$

For an equicontinuous system $\mathcal{E}_{F}=X$. Conversely, if $\mathcal{E}_{F}=X$ and if $X$ is compact, then $F$ is equicontinuous; this needs not be true in the non-compact case. A system $(X, F)$ is sensitive (to initial conditions), if

$$
(\exists \varepsilon>0)(\forall x \in X)(\forall \delta>0)\left(\exists y \in B_{\delta}(x)\right)(\exists n>0)\left(d\left(f^{n}(y), f^{n}(x)\right) \geq \varepsilon\right) .
$$

A sensitive system has no equicontinuous point. However, there exist systems with no equicontinuity points which are not sensitive. A system $(X, F)$ is positively expansive, if

$$
(\exists \varepsilon>0)(\forall x \neq y \in X)(\exists n \geq 0)\left(d\left(f^{n}(x), f^{n}(y)\right) \geq \varepsilon\right) .
$$

A positively expansive system on a perfect space is sensitive. A system $(X, F)$ is (topologically) transitive, if for any nonempty open sets $U, V \subseteq X$ there exists $n \geq 0$ such that $F^{-n}(U) \cap V \neq \emptyset$. If $X$ is perfect and if the system has a dense orbit, then it is transitive. Conversely, if $(X, F)$ is topologically transitive and if $X$ is compact, then $(X, F)$ has a dense orbit. A system $(X, F)$ is mixing, if for any nonempty open sets $U, V \subseteq X$ there exists $k>0$ such that for every $n \geq k$ we have $F^{-n}(U) \cap V \neq \emptyset$. An $\varepsilon$-chain (from $x_{0}$ to $x_{n}$ ) is a sequence of points $x_{0}, \ldots, x_{n} \in X$ such that $d\left(F\left(x_{i}\right), x_{i+1}\right)<\varepsilon$ for $0 \leq i<n$. A system $(X, F)$ is chain-transitive, if for any $\varepsilon>0$ and any $x, y \in X$ there exists an $\varepsilon$-chain from $x$ to $y$.

A strongly invariant closed set $Y \subseteq X$ is stable, if

$$
\forall \varepsilon>0, \exists \delta>0, \forall x \in X,(d(x, Y)<\delta \Longrightarrow \forall n>0, d(F(x), Y)<\varepsilon) .
$$

A strongly invariant closed stable set $Y \subseteq X$ is an attractor, if

$$
\exists \delta>0, \forall x \in X,\left(d(x, Y)<\delta \Longrightarrow \lim _{n \rightarrow \infty} d(F(x), Y)<\varepsilon\right) .
$$

A set $W \subseteq X$ is inward, if $F(\bar{W}) \subseteq W^{\circ}$. In compact spaces, attractors are exactly $\Omega$-limits $\Omega_{F}(W)=\bigcap_{n>0} F(W)$ of inward sets.

Theorem 1 (Knudsen [11]) Let $(X, F)$ be a DS and $Y \subseteq X$ a dense, $F$ invariant subset.

(1) $(X, F)$ is sensitive iff $(Y, F)$ is sensitive.

(2) $(X, F)$ is transitive iff $(Y, F)$ is transitive.

Recall that a space $X$ is separable, if it has a countable dense set.

Theorem 2 (Blanchard, Formenti, and Kůrka [3]) Let $(X, F)$ be a dynamical system on a non-separable space. If $(X, F)$ is transitive, then it is sensitive. 


\section{Cellular automata}

For a finite alphabet $A$, denote by $|A|$ the number of its elements, by $A^{*}:=$ $\bigcup_{n \geq 0} A^{n}$ the set of words over $A$, and by $A^{+}:=\bigcup_{n>0} A^{n}=A^{*} \backslash\{\lambda\}$ the set of nonempty words. The length of a word $u \in A^{n}$ is denoted by $|u|:=n$. We say that $u \in A^{*}$ is a subword of $v \in A^{*}(u \sqsubseteq v)$ if there exists $k$ such that $v_{k+i}=u_{i}$ for all $i<|u|$. We denote by $u_{[i, j)}=u_{i} \ldots u_{j-1}$ and $u_{[i, j]}=u_{i} \ldots u_{j}$ subwords of $u$ associated to intervals. We denote by $A^{\mathbb{Z}}$ the set of $A$-configurations, or doubly-infinite sequences of letters of $A$. For any $u \in A^{+}$we have a periodic configuration $u^{\infty} \in A^{\mathbb{Z}}$ defined by $\left(u^{\infty}\right)_{k|u|+i}=u_{i}$ for $k \in \mathbb{Z}$ and $0 \leq i<|u|$. The cylinder of a word $u \in A$ located at $l \in \mathbb{Z}$ is the set $[u]_{l}=\left\{x \in A^{\mathbb{Z}}\right.$ : $\left.x_{[l, l+|u|)}=u\right\}$. The cylinder set of a set of words $U \subseteq A^{+}$located at $l \in \mathbb{Z}$ is the set $[U]_{l}=\bigcup_{u \in U}[u]_{l}$.

A subshift is a nonempty subset $\Sigma \subseteq A^{\mathbb{Z}}$ such that there exists a set $D \subseteq A^{+}$of forbidden words and $\Sigma=\Sigma_{D}:=\left\{x \in A^{\mathbb{Z}}: \forall u \sqsubseteq x, u \notin D\right\}$. A subshift $\Sigma_{D}$ is of finite type (SFT), if $D$ is finite. A subshift is uniquely determined by its language

$$
\mathcal{L}(\Sigma):=\bigcup_{n \geq 0} \mathcal{L}^{n}(\Sigma), \quad \text { where } \quad \mathcal{L}^{n}(\Sigma):=\left\{u \in A^{n}: \exists x \in \Sigma, u \sqsubseteq x\right\} .
$$

A cellular automaton is a map $F: A^{\mathbb{Z}} \rightarrow A^{\mathbb{Z}}$ defined by $F(x)_{i}=$ $f\left(x_{[i-r, i+r]}\right)$, where $r \geq 0$ is a radius and $f: A^{2 r+1} \rightarrow A$ is a local rule. In particular the shift map $\sigma: A^{\mathbb{Z}} \rightarrow A^{\mathbb{Z}}$ is defined by $\sigma(x)_{i}:=x_{i+1}$. A local rule extends to the map $f: A^{*} \rightarrow A^{*}$ by $f(u)_{i}=f\left(u_{[i, i+2 r]}\right)$ so that $|f(u)|=\max \{|u|-2 r, 0\}$.

Definition 3 Let $F: A^{\mathbb{Z}} \rightarrow A^{\mathbb{Z}}$ be a $C A$.

(1) $A$ word $u \in A$ is $m$-blocking, if $|u| \geq m$ and there exists offset $d \leq|u|-m$ such that $\forall x, y \in[u]_{0}, \forall n>0, F^{n}(x)_{[d, d+m)}=F^{n}(y)_{[d, d+m)}$.

(2) A set $U \subseteq A^{+}$is spreading, if $[U]$ is $F$-invariant and there exists $n>0$ such that $F^{n}([U]) \subseteq \sigma^{-1}([U]) \cap \sigma([U])$.

The following results will be useful in the sequel.

Proposition 4 (Formenti, Kůrka [6]) Let $F: A^{\mathbb{Z}} \rightarrow A^{\mathbb{Z}}$ be a $C A$ and let $U \subseteq A^{+}$be an invariant set. Then $\Omega_{F}([U])$ is a subshift iff $U$ is spreading.

Theorem 5 (Hedlund [7]) Let $F: A^{\mathbb{Z}} \rightarrow A^{\mathbb{Z}}$ be a $C A$ with local rule $f$ : $A^{2 r+1} \rightarrow A$. Then $F$ is surjective iff $f: A^{*} \rightarrow A^{*}$ is surjective iff $\left|f^{-1}(u)\right|=$ $|A|^{2 r}$ for each $u \in A^{+}$.

\section{Submeasures}

A pseudometric on a set $X$ is a map $d: X \times X \rightarrow[0, \infty)$ which satisfies the following conditions:

1. $d(x, y)=d(y, x)$,

2. $d(x, z) \leq d(x, y)+d(y, z)$.

If moreover $d(x, y)>0$ for $x \neq y$, then we say that $d$ is a metric. There is a standard method to create pseudometrics from submeasures. A bounded submeasure (with bound $M \in \mathbb{R}^{+}$) is a map $\varphi: \mathcal{P}(\mathbb{Z}) \rightarrow[0, M]$ which satisfies the following conditions:

1. $\varphi(\emptyset)=0$,

2. $\varphi(U) \leq \varphi(U \cup V) \leq \varphi(U)+\varphi(V)$ for $U, V \subseteq \mathbb{Z}$.

A bounded submeasure $\varphi$ on $\mathbb{Z}$ defines a pseudometric $d_{\varphi}: A^{\mathbb{Z}} \times A^{\mathbb{Z}} \rightarrow[0, \infty)$ 
by $d_{\varphi}(x, y):=\varphi\left(\left\{i \in \mathbb{Z}: x_{i} \neq y_{i}\right\}\right)$. The Cantor, Besicovich and Weyl pseudometrics on $A^{\mathbb{Z}}$ are defined by the following submeasures:

$$
\begin{aligned}
\varphi_{\mathcal{C}}(U) & :=2^{-\min \{|i|: i \in U\}} \\
\varphi_{\mathcal{B}}(U) & :=\limsup _{l \rightarrow \infty} \frac{|U \cap[-l, l)|}{2 l} \\
\varphi_{\mathcal{W}}(U) & :=\limsup _{l \rightarrow \infty} \sup _{k \in \mathbb{Z}} \frac{|U \cap[k, k+l)|}{l}
\end{aligned}
$$

\section{The Cantor space}

The Cantor metric on $A^{\mathbb{Z}}$ is defined by

$$
d_{\mathcal{C}}(x, y)=2^{-k} \quad \text { where } \quad k=\min \left\{|i|: x_{i} \neq y_{i}\right\}
$$

so $d_{\mathcal{C}}(x, y)<2^{-k}$ iff $x_{[-k, k]}=y_{[-k, k]}$. We denote by $\mathcal{C}_{A}=\left(A^{\mathbb{Z}}, d_{\mathcal{C}}\right)$ the metric space of two-sided configurations with metric $d_{\mathcal{C}}$. The cylinders are clopen sets in $\mathcal{C}_{A}$. All Cantor spaces (with different alphabets) are homeomorphic. The Cantor space is compact, totally disconnected and perfect, and conversely, every space with these properties is homeomorphic to a Cantor space. Literature about CA dynamics in Cantor spaces is really huge. In this section, we just recall some results and definitions which will be used later.

Theorem 6 (Kůrka [12]) Let $\left(\mathcal{C}_{A}, F\right)$ be a $C A$ with radius $r$.

(1) $\left(\mathcal{C}_{A}, F\right)$ is almost equicontinuous iff there exists a r-blocking word for $F$

(2) $\left(\mathcal{C}_{A}, F\right)$ is equicontinuous iff all sufficiently long words are $r$-blocking.

Denote by $\mathcal{E}_{F}$ the set of equicontinuous points of $F$. The sets of equicontinuous directions and almost equicontinuous directions of a $\mathrm{CA}\left(\mathcal{C}_{A}, F\right)$ (see Sablik [18]) are defined by

$$
\begin{aligned}
\mathfrak{E}(F) & =\left\{\frac{p}{q}: p \in \mathbb{Z}, q \in \mathbb{N}^{+}, \mathcal{E}_{F^{q} \sigma^{p}}=A^{\mathbb{Z}}\right\}, \\
\mathfrak{A}(F) & =\left\{\frac{p}{q}: p \in \mathbb{Z}, q \in \mathbb{N}^{+}, \mathcal{E}_{F^{q} \sigma^{p}} \neq \emptyset\right\} .
\end{aligned}
$$

\section{The periodic space}

Definition 7 The periodic space $\mathcal{P}_{A}=\left\{x \in A^{\mathbb{Z}}: \exists n>0, \sigma^{n}(x)=x\right\}$ over an alphabet $A$ consists of shift periodic configurations with Cantor metric $d_{\mathcal{C}}$.

All periodic spaces (with different alphabets) are homeomorphic. The periodic space is not compact, but it is totally disconnected and perfect. It is dense in $\mathcal{C}_{A}$. If $\left(\mathcal{C}_{A}, F\right)$ is a $\mathrm{CA}$, Then $F\left(\mathcal{P}_{A}\right) \subseteq \mathcal{P}_{A}$. We denote by $F_{\mathcal{P}}: \mathcal{P}_{A} \rightarrow \mathcal{P}_{A}$ the restriction of $F$ to $\mathcal{P}_{A}$, so $\left(\mathcal{P}_{A}, F_{\mathcal{P}}\right)$ is a (non-compact) dynamical system. Every $F_{\mathcal{P}}$-orbit is finite, so every point $x \in \mathcal{P}_{A}$ is $F_{\mathcal{P}}$-eventually periodic.

Theorem 8 Let $F$ be a $C A$ over alphabet $A$.

(1) $\left(\mathcal{C}_{A}, F\right)$ is surjective iff $\left(\mathcal{P}_{A}, F_{\mathcal{P}}\right)$ is surjective.

(2) $\left(\mathcal{C}_{A}, F\right)$ is equicontinuous iff $\left(\mathcal{P}_{A}, F_{\mathcal{P}}\right)$ is equicontinuous.

(3) $\left(\mathcal{C}_{A}, F\right)$ is almost equicontinuous iff $\left(\mathcal{P}_{A}, F_{\mathcal{P}}\right)$ is almost equicontinuous.

(4) $\left(\mathcal{C}_{A}, F\right)$ is sensitive iff $\left(\mathcal{P}_{A}, F_{\mathcal{P}}\right)$ is sensitive.

(5) $\left(\mathcal{C}_{A}, F\right)$ is transitive iff $\left(\mathcal{P}_{A}, F_{\mathcal{P}}\right)$ is transitive. 
Proof: (1a) Let $F$ be surjective, let $y \in \mathcal{P}_{A}$ and $\sigma^{n}(y)=y$. There exists $z \in F^{-1}(y)$ and integers $i<j$ such that $z_{[i n r, i n r+r)}=z_{[j n r, j n r+r)}$. Then $x=\left(z_{[i n r, j n r)}\right)^{\infty} \in \mathcal{P}_{A}$ and $F_{\mathcal{P}}(x)=y$, so $F_{\mathcal{P}}$ is surjective.

(1b) Let $F_{\mathcal{P}}$ be surjective, and $u \in A^{+}$. Then $u^{\infty}$ has $F_{\mathcal{P}}$-preimage and therefore $u$ has preimage under the local rule. By Hedlund Theorem, $\left(\mathcal{C}_{A}, F\right)$ is surjective.

(2a) Since $\mathcal{P}_{A} \subset \mathcal{C}_{A}$, the equicontinuity of $F$ implies trivially the equicontinuity of $F_{\mathcal{P}}$.

(2b) Let $F_{\mathcal{P}}$ be equicontinuous. There exist $m>r$ such that if $x, y \in \mathcal{P}_{A}$ and $x_{[-m, m]}=y_{[-m, m]}$, then $F^{n}(x)_{[-r, r]}=F^{n}(y)_{[-r, r]}$ for all $n \geq 0$. We claim that all words of length $2 m+1$ are $(2 r+1)$-blocking with offset $m-r$. If not, then for some $x, y \in A^{\mathbb{Z}}$ with $x_{[-m, m]}=y_{[-m, m]}$, there exists $n>0$ such that $F^{n}(x)_{[-r, r]} \neq F^{n}(y)_{[-r, r]}$. For periodic configurations $x^{\prime}=\left(x_{[-m-n r, m+n r]}\right)^{\infty}$, $y^{\prime}=\left(y_{[-m-n r, m+n r]}\right)^{\infty}$ we get $F^{n}\left(x^{\prime}\right)_{[-r, r]} \neq F^{n}\left(y^{\prime}\right)_{[-r, r]}$ contradicting the assumption. By Theorem $6, F$ is $\mathcal{C}$-equicontinuous.

(3a) If $\left(\mathcal{C}_{A}, F\right)$ is almost equicontinuous, then there exists a $r$-blocking word $u$ and $u^{\infty} \in \mathcal{P}_{A}$ is an equicontinuous configuration for $\left(\mathcal{P}_{a}, F_{\mathcal{P}}\right)$.

(3b) The proof is analogous as (2b).

(4) and (5) follow from the Theorem 1 of Knudsen.

\section{The Toeplitz space}

Definition 9 Let $A$ be an alphabet

(1) The Besicovitch pseudometric on $A^{\mathbb{Z}}$ is defined by

$$
d_{\mathcal{B}}(x, y)=\limsup _{l \rightarrow \infty} \frac{\left|\left\{j \in[-l, l): x_{j} \neq y_{j}\right\}\right|}{2 l}
$$

(2) The Weyl pseudometric on $A^{\mathbb{Z}}$ is defined by

$$
d_{\mathcal{W}}(x, y)=\limsup _{l \rightarrow \infty} \max _{k \in \mathbb{Z}} \frac{\left|\left\{j \in[k, k+l): x_{j} \neq y_{j}\right\}\right|}{l}
$$

Clearly $d_{\mathcal{B}}(x, y) \leq d_{\mathcal{W}}(x, y)$ and

$$
\begin{aligned}
d_{\mathcal{B}}(x, y)<\varepsilon & \Longleftrightarrow \quad \exists l_{0} \in \mathbb{N}, \forall l \geq l_{0},\left|\left\{j \in[-l, l]: x_{j} \neq y_{j}\right\}\right|<(2 l+1) \varepsilon . \\
d_{\mathcal{W}}(x, y)<\varepsilon & \Longleftrightarrow \quad \exists l_{0} \in \mathbb{N}, \forall l \geq l_{0}, \forall k \in \mathbb{Z},\left|\left\{j \in[k, k+l): x_{j} \neq y_{j}\right\}\right|<l \varepsilon
\end{aligned}
$$

Both $d_{\mathcal{B}}$ and $d_{\mathcal{W}}$ are symmetric and satisfy the triangle inequality, but they are not metrics. Distinct configurations $x, y \in A^{\mathbb{Z}}$ can have zero distance. We construct a set of regular quasi-periodic configurations, on which $d_{\mathcal{B}}$ and $d_{\mathcal{W}}$ coincide and are metrics.

\section{Definition 10}

(1) The period of $k \in \mathbb{Z}$ in $x \in A^{\mathbb{Z}}$ is $r_{k}(x):=\inf \left\{p>0: \forall n \in \mathbb{Z}, x_{k+n p}=\right.$ $\left.x_{k}\right\}$. We set $r_{k}(x)=\infty$ if the defining set is empty.

(2) $x \in A^{\mathbb{Z}}$ is quasi-periodic, if $r_{k}(x)<\infty$ for all $k \in \mathbb{Z}$.

(3) $A$ periodic structure for a quasi-periodic configuration $x$ is a sequence of positive integers $\mathbf{p}=\left(p_{i}\right)_{i<|\mathbf{p}| \leq \infty}$, such that $p_{i} \mid p_{i+1} \quad\left(p_{i}\right.$ divides $\left.p_{i+1}\right)$, and for every $k \in \mathbb{Z}, r_{k}(x) \mid p_{i}$ for some $i$.

(3) A quasi-periodic configuration $x \in A^{\mathbb{Z}}$ is regular, if for some periodic structure $\mathbf{p}$ of $x$ we have $\lim _{i \rightarrow \infty} q_{i}(x) / p_{i}=0$, where $q_{i}(x):=\mid\{k \in$ $\left.\left[0, p_{i}\right)_{i}: r_{k}(x) \not p_{i}\right\} \mid\left(r_{k}(x)\right.$ does not divide $\left.p_{i}\right)$. 
Clearly every $\sigma$-periodic configuration is quasi-periodic and has a finite periodic structure.

\section{Proposition 11}

(1) If $x, y$ are regular quasi-periodic configurations, then $d_{\mathcal{W}}(x, y)=d_{\mathcal{B}}(x, y)$.

(2) If $x \neq y$ are quasi-periodic configurations, then $d_{\mathcal{W}}(x, y) \geq d_{\mathcal{B}}(x, y)>0$.

Proof: (1) We must show $d_{\mathcal{W}}(x, y) \leq d_{\mathcal{B}}(x, y)$. Let $\mathbf{p}^{x}, \mathbf{p}^{y}$ be the periodic structures for $x$ and $y$ and let $p_{i}=k_{i}^{x} p_{i}^{x}=k_{i}^{y} p_{i}^{y}$ be the lowest common multiple of $p_{i}^{x}$ and $p_{i}^{y}$. Then $\mathbf{p}=\left(p_{i}\right)_{i}$ is a periodic structure for both $x$ and $y$. For each $i>0$ and for each $k \in \mathbb{Z}$ we have

$$
\begin{aligned}
\mid\left\{j \in \left[k-p_{i}, k+\right.\right. & \left.\left.p_{i}\right): x_{j} \neq y_{j}\right\}\left|\leq 2 k_{i}^{x} q_{i}^{x}+2 k_{i}^{y} q_{i}^{y}+\right|\left\{j \in\left[-p_{i}, p_{i}\right): x_{j} \neq y_{j}\right\} \mid \\
d_{\mathcal{W}}(x, y) & \leq \lim _{i \rightarrow \infty} \max _{k \in \mathbb{Z}}\left|\left\{j \in\left[k-p_{i}, k+p_{i}\right): x_{j} \neq y_{j}\right\}\right| \\
& \leq \lim _{i \rightarrow \infty}\left(\frac{2 k_{i}^{x} q_{i}^{x}}{2 k_{i}^{x} p_{i}^{x}}+\frac{2 k_{i}^{y} q_{i}^{y}}{2 k_{i}^{y} p_{i}^{y}}+\frac{\left|\left\{j \in\left[-p_{i}, p_{i}\right): x_{j} \neq y_{j}\right\}\right|}{2 p_{i}}\right) \\
& =d_{\mathcal{B}}(x, y)
\end{aligned}
$$

(2) Since $x \neq y$, there exists $i$ such that for some $k \in\left[0, p_{i}\right)$ and for all $n \in \mathbb{Z}$ we have $x_{k+n p_{i}}=x_{k} \neq y_{k}=y_{k+n p_{i}}$. It follows $d_{\mathcal{B}}(x, y) \geq 1 / p_{i}$.

Definition 12 The Toeplitz space $\mathcal{T}_{A}$ over $A$ consists of all regular quasiperiodic configurations with metric $d_{\mathcal{B}}=d_{\mathcal{W}}$.

Toeplitz sequences are constructed by filling in periodic parts successively. For an alphabet $A$ put $\widetilde{A}=A \cup\{*\}$.

\section{Definition 13}

(1) The $p$-skeleton $S_{p}(x) \in \widetilde{A}^{\mathbb{Z}}$ of $x \in A^{\mathbb{Z}}$ is defined by

$$
S_{p}(x)_{k}= \begin{cases}x_{k} & \text { if } \forall n \in \mathbb{Z}, x_{k+n p}=x_{k} \\ * & \text { otherwise }\end{cases}
$$

(2) The sequence of gaps of $x \in \widetilde{A}^{\mathbb{Z}}$ is the unique increasing integer sequence $\left(t_{i}\right)_{a<i<b}$ such that $x_{t_{i}}=*, x_{k} \neq *$ for $t_{i}<k<t_{i+1}$ and $t_{-1}<0 \leq t_{0}$.

(3) Let $x, y \in \widetilde{A}^{\mathbb{Z}}$ and let $\left(t_{i}\right)$ be the sequence of gaps of $x$. The amalgamation $T(x, y) \in \widetilde{A}^{\mathbb{Z}}$ of $x, y$ is

$$
T(x, y)_{i}= \begin{cases}x_{i} & \text { if } x_{i} \neq * \\ y_{j} & \text { if } x_{i}=* \& i=t_{j}\end{cases}
$$

The $p$-skeleton is $p$-periodic. If $p$ is its smallest period, we say that $p$ is an essential period of $x$. The sequence of gaps may be two-way infinite (then $a=-\infty, b=\infty)$, one-way infinite $(a=-\infty, b<\infty$ or $-\infty<a, b<\infty)$, finite $(-\infty<a<b<\infty)$ or even empty when $x \in A^{\mathbb{Z}}$. If it is nonempty then it must be defined at least on -1 or 0 .

Proposition 14 Let $\mathbf{2}:=\{0,1\}$ be the binary alphabet and $[0,1]$ the real unit interval (with standard metric). There exists an isometry $f:[0,1] \rightarrow \mathcal{T}_{2}$ such that $f(0)=0^{\infty}$ and $f(1)=1^{\infty}$.

Proof: Consider a map $h: \mathbf{2}^{*} \rightarrow \widetilde{\mathbf{2}}^{\mathbb{Z}}$ defined by $h(\lambda)=*^{\infty}, h(0)=(0 *)^{\infty}$, $h(1)=(* 1)^{\infty}, h\left(x_{0} \ldots x_{n-1} x_{n}\right)=T\left(h\left(x_{0} \ldots x_{n-1}\right), h\left(x_{n}\right)\right)$. Thus 


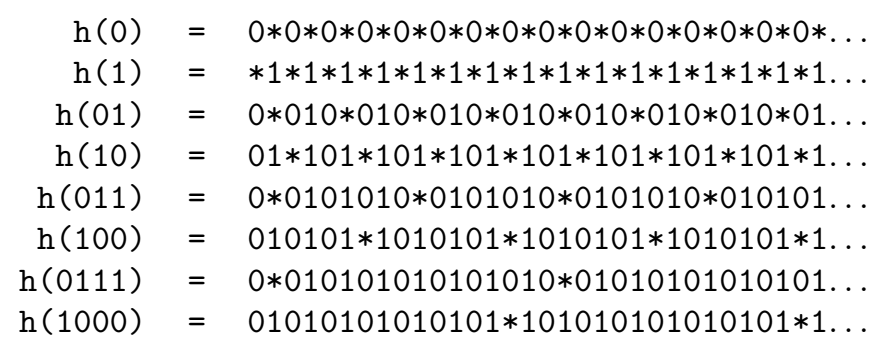

For $x \in \mathbf{2}^{\mathbb{N}}$, the limit (in the Cantor topology) $h(x)=\lim _{n \rightarrow \infty} h\left(x_{0}, \ldots x_{n}\right)$ exists. If no suffix of $x$ is $1^{\infty}$, then $h(x) \in \mathbf{2}^{\mathbb{Z}}$, otherwise $h(x)$ contains exactly one star and we replace it by 1 . Thus for each $x \in \mathbf{2}^{\mathbb{N}}, h(x) \in \mathbf{2}^{\mathbb{Z}}$ is a regular quasi-periodic sequence. It can be verified directly that $h(0111 \ldots)=(01)^{\infty}=$ $h(1000 \ldots)$. Using an easily verifiable formula $T(x, T(y, z))=T(T(x, y), z)$, we get

$$
\begin{aligned}
h\left(x_{0} \ldots x_{n} 01^{\infty}\right) & =T\left(h\left(x_{0} \ldots x_{n}\right), h\left(01^{\infty}\right)=T\left(h\left(x_{0} \ldots x_{n}\right), h\left(10^{\infty}\right)\right)\right. \\
& =h\left(x_{0} \ldots x_{n} 10^{\infty}\right)
\end{aligned}
$$

For a real number $x \in[0,1]$ with binary expansion $x=\sum_{i=0}^{\infty} x_{i} 2^{-i-1}$ put $f(x)=h\left(x_{0} x_{1} x_{2} x_{3} \ldots\right)$. If $2^{n} x$ is an integer for some $n$, then $x$ has two binary expansions, and $f(x)$ is the same for both expansions. If $|x-y| \leq 2^{-m}$, then $x_{[0, m)}=y_{[0, m)}$; therefore $d_{\mathcal{W}}(f(x), f(y)) \leq 2^{-m}$ and $f:[0,1] \rightarrow \overline{\mathcal{T}}_{A}$ is continuous. For dyadic numbers $x, y$ of the form $k / 2^{m}$ we verify $d_{\mathcal{B}}(f(x), f(y))=$ $|x-y|$, so $f$ is an isometry.

Proposition 15 The Toeplitz space $\mathcal{T}_{A}$ of regular quasi-periodic sequences is pathwise connected and infinite-dimensional.

Proof: Assume that the alphabet $A$ contains letters 0,1 and consider the map $f:[0,1] \rightarrow A^{\mathbb{Z}}$ from Proposition 14. For $a \in A$ set $a \cdot 0=0, a \cdot 1=a$. Given $u \in \mathbf{2}^{\mathbb{Z}}$ the map $g_{u}:[0,1] \rightarrow \mathbf{2}^{\mathbb{Z}}$ defined by $g_{u}(x)_{i}=u_{i} f(x)_{i}$ is continuous, $g_{u}(0)=0$ and $g_{u}(1)=u$. Thus $\mathcal{T}_{A}$ is pathwise connected. To show that $\mathcal{T}_{A}$ is infinite-dimensional, construct for any $n$ an embedding $f_{n}:[0,1]^{n} \rightarrow X_{W}$ of an $n$-dimensional cube by

$$
f_{n}\left(x_{1}, \ldots, x_{n}\right)=f\left(x_{1}\right)_{0} \ldots f\left(x_{n}\right)_{0} f\left(x_{1}\right)_{1} \ldots f\left(x_{n}\right)_{1} \ldots
$$

Thus $\mathcal{T}_{A}$ is at least $n$-dimensional and therefore infinite-dimensional.

Proposition 16 Let $F: A^{\mathbb{Z}} \rightarrow A^{\mathbb{Z}}$ be a $C A$ with radius $r$.

(1) If $x \in A^{\mathbb{Z}}$ is a quasi-periodic with periodic structure $\mathbf{p}$, then $F(x)$ is quasiperiodic with periodic structure $\mathbf{p}$.

(2) If $x$ is regular quasi-periodic, then $F(x)$ is regular.

Proof: (1) For $k \in \mathbb{Z}$ denote by $m:=\min \left\{i: \forall j \in[k-r, k+r], r_{j}(x) \mid p_{i}\right\}$. Then $p_{m}$ is a period of $F(x)_{k}$.

(2) We have

$$
\begin{aligned}
q_{i}(F(x)) & :=\left|\left\{k<p_{i}: r_{k}(F(x)) \chi p_{i}\right\}\right| \\
& \leq(2 r+1) \cdot\left|\left\{k<p_{i}: r_{k}(x) \backslash p_{i}\right\}\right| \\
& =(2 r+1) \cdot q_{i}(x)
\end{aligned}
$$

so

$$
\lim _{i \rightarrow \infty} \frac{q_{i}(F(x))}{p_{i}} \leq(2 r+1) \cdot \lim _{i \rightarrow \infty} \frac{q_{i}(x)}{p_{i}}=0
$$

For a CA $F$ we denote by $F_{\mathcal{T}}$ the restriction of $F$ to $\mathcal{T}_{A}$. 
Theorem 17 Let $F$ be a $C A$.

(1) $\left(\mathcal{C}_{A}, F\right)$ is surjective iff $\left(\mathcal{T}_{A}, F_{\mathcal{T}}\right)$ is surjective.

(2) If $\mathfrak{A}(F) \neq \emptyset$, then $\left(\mathcal{T}_{A}, F_{\mathcal{T}}\right)$ is almost equicontinuous.

(3) if $\mathfrak{E}(F) \neq \emptyset$, then $\left(\mathcal{T}_{A}, F_{\mathcal{T}}\right)$ is equicontinuous.

(4) If $\left(\mathcal{C}_{A}, F\right)$ is chain-transitive, then $\left(\mathcal{T}_{A}, F_{\mathcal{T}}\right)$ is chain-transitive.

(5) $\left(\mathcal{T}_{A}, F_{\mathcal{T}}\right)$ is injective iff it is surjective.

Proof: (1) The proof is the same as in Theorem 8(1)

(2) Assume first that $F$ is almost equicontinuous, so there exists $m>r$ and $u \in$ $A^{2 m+1}$ such that for any $x, y \in[u]_{-m}, F^{n}(x)_{[-r, r]}=F^{n}(y)_{[-r, r]}$ for all $n>0$. We show that $u^{\infty}$ is $\mathcal{T}$-equicontinuous. For a given $\varepsilon>0$ set $\delta=\varepsilon /(4 m-2 r+1)$. If $d_{\mathcal{T}}(y, x)<\delta$, then there exists $l_{0}$ such that for all $l \geq l_{0}, \mid\left\{i \in[-l, l]: x_{i} \neq\right.$ $\left.y_{i}\right\} \mid<(2 l+1) \delta$. For $k(2 m+1) \leq j<(k+1)(2 m+1), F^{n}(y)_{j}$ can differ from $F^{n}(x)_{j}$ only if $y$ differs from $x$ in some $i \in[k(2 m+1)-(m-r),(k+1) m+(m-r))$ Thus a change $x_{i} \neq y_{i}$ can cause at most $2 m+1+2(m-r)=4 m-2 r+1$ changes $F^{n}(y)_{j} \neq F^{n}(x)_{j}$. We get

$$
\left|\left\{i \in[-l, l): F^{n}(x)_{i} \neq F^{n}(y)_{i}\right\}\right| \leq 2 l \delta(4 m-2 r+1) \leq 2 l \varepsilon
$$

This shows that $F_{\mathcal{T}}$ is almost equicontinuous. In the general case that $\mathfrak{A}(F) \neq$ $\emptyset$, we get that $F_{\mathcal{T}}^{q} \sigma^{p}$ is almost equicontinuous for some $p \in \mathbb{Z}, q \in \mathbb{N}^{+}$. Since $\sigma$ is $\mathcal{T}$-equicontinuous, $F_{\mathcal{T}}^{q}$ is almost equicontinuous and therefore $\left(\mathcal{T}_{A}, F_{\mathcal{T}}\right)$ is almost equicontinuous.

(3) The proof is the same as in (2) with the only modification that all $u \in A^{m}$ are $(2 r+1)$-blocking.

(4) The proof of Proposition 8 from [3] works in this case too.

(5) The proof of Proposition 12 of [2] works in this case also.

\section{The Besicovitch space}

On $A^{\mathbb{Z}}$ we have an equivalence $x \approx_{\mathcal{B}} y$ iff $d_{\mathcal{B}}(x, y)=0$. Denote by $\mathcal{B}_{A}$ the set of equivalence classes of $\approx_{B}$ and by $\pi_{B}: A^{\mathbb{Z}} \rightarrow \mathcal{B}_{A}$ the projection. The factor of $d_{\mathcal{B}}$ is a metric on $\mathcal{B}_{A}$. This is the Besicovitch space on alphabet $A$. Using prefix codes, it can be shown that every two Besicovitch spaces (with different alphabets) are homeomorphic. By Proposition 11 each equivalence class contains at most one quasi-periodic sequence.

Proposition $18 \mathcal{T}_{A}$ is dense in $\mathcal{B}_{A}$

The proof of Proposition 9 of [2] works also for regular quasi-periodic sequences.

Theorem 19 (Blanchard, Formenti and Kůrka [3])

The Besicovitch space is pathwise connected, infinite-dimensional, homogenous and complete. It is neither separable nor locally compact.

The properties of path-connectedness and infinite dimensionality is proved analogously as in Proposition 15. To prove that $\mathcal{B}_{A}$ is neither separable nor locally compact, Sturmian configurations have been used in [3]. The completeness of $\mathcal{B}_{A}$ has been proved by Marcinkiewicz [16]. Every cellular automaton $F: A^{\mathbb{Z}} \rightarrow A^{\mathbb{Z}}$ is uniformly continuous with respect to $d_{\mathcal{B}}$, so it preserves the equivalence $\approx_{B}$. If $d_{\mathcal{B}}(x, y)=0$, then $d_{\mathcal{B}}(F(x), F(y))=0$. Thus a cellular automaton $F$ defines a uniformly continuous map $F_{\mathcal{B}}: \mathcal{B}_{A} \rightarrow \mathcal{B}_{A}$.

Theorem 20 (Blanchard, Formenti and Kůrka [3]) Let $F$ be a $C A$ on $A$. 
(1) $\left(\mathcal{C}_{A}, F\right)$ is surjective iff $\left(\mathcal{B}_{A}, F_{\mathcal{B}}\right)$ is surjective.

(2) If $\mathfrak{A}(F) \neq \emptyset$ then $\left(\mathcal{B}_{A}, F_{\mathcal{B}}\right)$ is almost equicontinuous.

(3) if $\mathfrak{E}(F) \neq \emptyset$, then $\left(\mathcal{B}_{A}, F_{\mathcal{B}}\right)$ is equicontinuous.

(4) If $\left(\mathcal{B}_{A}, F_{\mathcal{B}}\right)$ is sensitive, then $\left(\mathcal{C}_{A}, F\right)$ is sensitive.

(5) No cellular automaton $\left(\mathcal{B}_{A}, F_{\mathcal{B}}\right)$ is positively expansive.

(6) If $\left(\mathcal{C}_{A}, F\right)$ is chain-transitive, then $\left(\mathcal{B}_{A}, F_{\mathcal{B}}\right)$ is chain-transitive.

\section{Theorem 21 (Blanchard, Cervelle and Formenti [2])}

(1) No $C A\left(\mathcal{B}_{A}, F_{\mathcal{B}}\right)$ is transitive.

(2) $A C A\left(\mathcal{B}_{A}, F_{\mathcal{B}}\right)$ has either a unique fixed point and no other periodic point, or it has uncountably many periodic points.

(3) If a surjective $C A$ has a blocking word, then the set of its $F_{\mathcal{B}}$-periodic points is dense in $\mathcal{B}_{A}$.

\section{The generic space}

For a configuration $x \in A^{\mathbb{Z}}$ and word $v \in A^{+}$set

$$
\begin{aligned}
& \underline{\Phi}_{v}(x)=\liminf _{n \rightarrow \infty}\left|\left\{i \in[-n, n): x_{[i, i+|v|)}=v\right\}\right| / 2 n, \\
& \bar{\Phi}_{v}(x)=\limsup _{n \rightarrow \infty}\left|\left\{i \in[-n, n): x_{[i, i+|v|)}=v\right\}\right| / 2 n .
\end{aligned}
$$

For every $v \in A^{*}, \underline{\Phi}_{v}, \bar{\Phi}_{v}: A^{\mathbb{Z}} \rightarrow[0,1]$ are continuous in the Besicovitch topology. In fact we have

$$
\begin{aligned}
\left|\Phi_{v}(x)-\bar{\Phi}_{v}(y)\right| & \leq d_{\mathcal{B}}(x, y) \cdot|v|, \\
\left|\underline{\Phi}_{v}(x)-\underline{\Phi}_{v}(y)\right| & \leq d_{\mathcal{B}}(x, y) \cdot|v|
\end{aligned}
$$

Define the generic space (over the alphabet $A$ ) as

$$
\mathcal{G}_{A}=\left\{x \in A^{\mathbb{Z}}: \forall v \in A^{*}, \underline{\Phi}_{v}(x)=\bar{\Phi}_{v}(x)\right\}
$$

It is a closed subspace of $\mathcal{B}_{A}$. For $v \in A^{*}$ denote by $\Phi_{v}: \mathcal{G}_{A} \rightarrow[0,1]$ the common value of $\underline{\Phi}$ and $\bar{\Phi}$.

Using prefix codes, one can show that all generic spaces (with different alphabets) are homeomorhic. The generic space contains all uniquely ergodic subshifts, in particular all Sturmian sequences and all regular Toeplitz sequences. Thus the proofs in Blanchard Formenti and Kurka [3] can be applied to the generic space too. In particular the generic space is homogenous. If we regard the alphabet $A=\{0, \ldots, m-1\}$ as the group $\mathbb{Z}_{m}=\mathbb{Z} / m \mathbb{Z}$, then for every $x \in \mathcal{G}_{A}$ there is an isometry $H_{x}: \mathcal{G}_{A} \rightarrow \mathcal{G}_{A}$ defined by $H_{x}(y)=x+y$. Moreover, $\mathcal{G}_{A}$ is pahtwise connected, infinite-dimensional and complete (as a closed subspace the full Besicovitch space). It is neither separable nor locally compact. If $F: A^{\mathbb{Z}} \rightarrow A^{\mathbb{Z}}$ is a cellular automaton, then $F\left(\mathcal{G}_{A}\right) \subseteq \mathcal{G}_{A}$. Thus, the restriction of $F_{\mathcal{B}}$ to $\mathcal{G}_{A}$ defines a dynamical system $\left(\mathcal{G}_{A}, F_{\mathcal{G}}\right)$.

Theorem 22 Let $F: A^{\mathbb{Z}} \rightarrow A^{\mathbb{Z}}$ be a $C A$.

(1) $\left(\mathcal{C}_{A}, F\right)$ is surjective iff $\left(\mathcal{G}_{A}, F_{\mathcal{G}}\right)$ is surjective.

(2) If $\mathfrak{A}(F) \neq \emptyset$, then $\left(\mathcal{G}_{A}, F_{\mathcal{G}}\right)$ is almost equicontinuous.

(3) if $\mathcal{E}(F) \neq \emptyset$, then $\left(\mathcal{G}_{A}, F_{\mathcal{G}}\right)$ is equicontinuous.

(4) If $\left(\mathcal{G}_{A}, F_{\mathcal{G}}\right)$ is sensitive, then $\left(\mathcal{C}_{A}, F\right)$ is sensitive.

(5) If $F$ is $\mathcal{C}$-chain transitive, then $F$ is $\mathcal{G}$-chain transitive.

The proofs are the same as the proofs of corresponding properties in [3]. 


\section{The space of measures}

By a measure we mean a Borel shift-invariant probability measure on the Cantor space $A^{\mathbb{Z}}$ (see Pivato [17]). This is a countably additive function $\mu$ on the Borel sets of $A^{\mathbb{Z}}$ which assigns 1 to the full space and satisfies $\mu(U)=$ $\mu\left(\sigma^{-1}(U)\right)$. A measure on $A^{\mathbb{Z}}$ is determined by its values on cylinders $\mu(u):=$ $\mu\left([u]_{n}\right)$ which does not depend on $n \in \mathbb{Z}$. Thus a measure can be identified with a map $\mu: A^{*} \rightarrow[0,1]$ subject to bilateral Kolmogorov compatibility conditions

$$
\sum_{a \in A} \mu(u a)=\sum_{a \in A} \mu(a u)=\mu(u), \quad \mu(\lambda)=1
$$

Define the distance of two measures

$$
d_{\mathcal{M}}(\mu, \nu):=\sum_{u \in A^{+}}|\mu(u)-\nu(u)| \cdot|A|^{-2|u|}
$$

This is a metric which yields the topology of weak* convergence on the compact space $\mathcal{M}_{A}:=\mathcal{M}_{\sigma}\left(A^{\mathbb{Z}}\right)$ of shift-invariant Borel probability measures. A CA $F: A^{\mathbb{Z}} \rightarrow A^{\mathbb{Z}}$ with local rule $f$ determines a continuous and affine map $F_{\mathcal{M}}$ : $\mathcal{M}_{A} \rightarrow \mathcal{M}_{A}$ by

$$
\left(F_{\mathcal{M}}(\mu)\right)(u)=\sum_{v \in f^{-1}(u)} \mu(v)
$$

Moreover $F$ and $F \sigma$ determine the same dynamical system on $\mathcal{M}_{A}: F_{\mathcal{M}}=$ $(F \sigma)_{\mathcal{M}}$.

For $x \in \mathcal{G}_{A}$ denote by $\Phi^{x}: A^{*} \rightarrow[0,1]$ the function $\Phi^{x}(v)=\Phi_{v}(x)$. For every $x \in \mathcal{G}_{A}, \Phi^{x}$ is a shift-invariant Borel probability measure. The map $\Phi: \mathcal{G}_{A} \rightarrow \mathcal{M}_{A}$ is continuous with respect to the Besicovich and weak* topologies. In fact we have

$$
\begin{aligned}
d_{\mathcal{M}}\left(\Phi^{x}, \Phi^{y}\right) & \leq d_{\mathcal{B}}(x, y) \sum_{u \in A^{+}}|u| \cdot|A|^{-2|u|}=d_{\mathcal{B}}(x, y) \sum_{n>0} n \cdot|A|^{-n} \\
& =d_{\mathcal{B}}(x, y) \cdot|A| /(|A|-1)^{2}
\end{aligned}
$$

By a theorem of Kamae [10], $\Phi$ is surjective. Every shift-invariant Borel probability measure has a generic point. It follows from the Ergodic Theorem that if $\mu$ is a $\sigma$-invariant measure, then $\mu\left(\mathcal{G}_{A}\right)=1$ and for every $v \in A^{*}$, the measure of $v$ is the integral of its density $\Phi_{v}$,

$$
\mu(v)=\int \Phi_{v}(x) d \mu
$$

If $F$ is a $\mathrm{CA}$, we have a commutative diagram $\Phi F_{\mathcal{G}}=F_{\mathcal{M}} \Phi$.

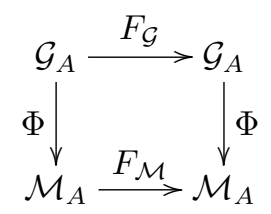

Theorem 23 Let $F$ be a $C A$ over $A$.

(1) $\left(\mathcal{C}_{A}, F\right)$ is surjective iff $\left(\mathcal{M}_{A}, F_{\mathcal{M}}\right)$ is surjective.

(2) If $\left(\mathcal{G}_{A}, F_{\mathcal{G}}\right)$ has dense set of periodic points, then $\left(\mathcal{M}_{A}, F_{\mathcal{M}}\right)$ has dense set of periodic points.

(3) If $\mathcal{A}(F) \neq \emptyset$, then $\left(\mathcal{M}_{A}, F_{\mathcal{M}}\right)$ is almost equicontinuous.

(4) If $\mathfrak{E}(F) \neq \emptyset$, then $\left(\mathcal{M}_{A}, F_{\mathcal{M}}\right)$ is equicontinuous. 
Proof: (1) See Kưrka [14] for a proof.

(2) This holds since $\left(\mathcal{M}_{A}, F_{\mathcal{M}}\right)$ is a factor of $\left(\mathcal{G}_{A}, F_{\mathcal{G}}\right)$.

(3) It suffices to prove the claim for the case that $F$ is almost equicontinuous. In this case there exists a blocking word $u \in A^{+}$and the Dirac measure $\delta_{u}$ defined by

$$
\delta_{u}(v)=\left\{\begin{array}{lll}
1 /|u| & \text { if } & v \sqsubseteq u \\
0 & \text { if } \quad v \nsubseteq u
\end{array}\right.
$$

is equicontinuous for $\left(\mathcal{M}_{A}, F_{\mathcal{M}}\right)$.

(4) If $\left(\mathcal{C}_{A}, F\right)$ is equicontinuous, then all sufficiently long words are blocking and there exists $d>0$ such that for all $n>0$, and for all $x, y \in A^{\mathbb{Z}}$ such that $x_{[-n-d, n+d]}=y_{[-n-d, n+d]}$ we have $F^{k}(x)_{[-n, n]}=F^{k}(y)_{[-n, n]}$ for all $k>0$. Thus there are maps $g_{k}: A^{*} \rightarrow A^{*}$ such that $\left|g_{k}(u)\right|=\max \{|u|-2 d, 0\}$ and for every $x \in A^{\mathbb{Z}}$ we have $F^{k}(x)_{[-n, n]}=F_{k}\left(x_{[-n-k d, n+k d]}\right)=g_{k}\left(x_{[-n-d, n+d]}\right)$, where $f$ is the local rule for $F$. We get

$$
\begin{aligned}
d_{\mathcal{M}}\left(F_{\mathcal{M}}^{k}(\mu), F_{\mathcal{M}}^{k}(\nu)\right) & =\sum_{n=1}^{\infty} \sum_{u \in A^{n}}\left|\sum_{v \in f^{-k}(u)}(\mu(v)-\nu(v))\right| \cdot|A|^{-2 n} \\
& =\sum_{n=1}^{\infty} \sum_{u \in A^{n}}\left|\sum_{v \in g_{k}^{-1}(u)}(\mu(v)-\nu(v))\right| \cdot|A|^{-2 n} \\
& \leq \sum_{n=1}^{\infty} \sum_{v \in A^{n+2 d}}|\mu(v)-\nu(v)| \cdot|A|^{-2 n} \\
& \leq|A|^{4 d} \cdot d_{\mathcal{M}}(\mu, \nu)
\end{aligned}
$$

\section{The Weyl space}

Define the following equivalence relation on $A^{\mathbb{Z}}: x \approx_{\mathcal{W}} y$ iff $d_{\mathcal{W}}(x, y)=0$. Denote by $\mathcal{W}_{A}$ the set of equivalence classes of $\approx_{W}$ and by $\pi_{W}: A^{\mathbb{Z}} \rightarrow \mathcal{W}_{A}$ the projection. The factor of $d_{\mathcal{W}}$ is a metric on $\mathcal{W}_{A}$. This is the Weyl space on alphabet $A$. Using prefix codes, it can be shown that every two Weyl spaces (with different alphabets) are homeomorphic. The Toeplitz space is not dense in the Weyl space (see Blanchard, Cervelle and Formenti [2]).

\section{Theorem 24 (Blanchard, Formenti and Kůrka [3])}

The Weyl space is pathwise connected, infinite-dimensional and homogenous. It is neither separable nor locally compact. It is not complete.

Every cellular automaton $F: A^{\mathbb{Z}} \rightarrow A^{\mathbb{Z}}$ is continuous with respect to $d_{\mathcal{W}}$, so it preserves the equivalence $\approx_{W}$. If $d_{\mathcal{W}}(x, y)=0$, then $d_{\mathcal{W}}(F(x), F(y))=0$. Thus a cellular automaton $F$ defines a continuous map $F_{\mathcal{W}}: \mathcal{W}_{A} \rightarrow \mathcal{W}_{A}$. The shift map $\sigma: \mathcal{W}_{A} \rightarrow \mathcal{W}_{A}$ is again an isometry, so in $\mathcal{W}_{A}$ many topological properies are preserved if $F$ is composed with a power of the shift. This is true for example for equicontinuity, almost continuity and sensitivity. If $\pi: \mathcal{W}_{A} \rightarrow \mathcal{B}_{A}$ is the (continuous) projection and $F$ a $\mathrm{CA}$, then the following diagram commutes.

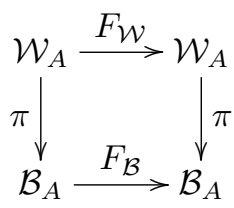




\section{Theorem 25 (Blanchard, Formenti and Kůrka [3])}

Let $F$ be a $C A$ on $A$.

(1) $\left(\mathcal{C}_{A}, F\right)$ is surjective iff $\left(\mathcal{W}_{A}, F_{\mathcal{W}}\right)$ is surjective.

(2) If $\mathfrak{A}(F) \neq \emptyset$, then $\left(\mathcal{W}_{A}, F_{\mathcal{W}}\right)$ is almost equicontinuous.

(3) if $\mathcal{E}(F) \neq \emptyset$, then $\left(\mathcal{W}_{A}, F_{\mathcal{W}}\right)$ is equicontinuous.

(4) If $\left(\mathcal{C}_{A}, F\right)$ is chain-transitive, then $\left(\mathcal{W}_{A}, F_{\mathcal{W}}\right)$ is chain-transitive.

Theorem 26 (Blanchard, Cervelle and Formenti [2])

No $C A$ is $\left(\mathcal{W}_{A}, F_{\mathcal{W}}\right)$ is transitive.

Theorem 27 Let $\Sigma$ be a subshift attractor of finite type for $F$ (in the Cantor space). Then there exists $\delta>0$ such that for every $x \in \mathcal{W}_{A}$ satisfying $d_{\mathcal{W}}(x, \Sigma)<\delta, F^{n}(x) \in \Sigma$ for some $n>0$.

Thus a subshift attractor of finite type is a $\mathcal{W}$-attractor. Example 2 shows that it need not be $\mathcal{B}$-attractor. Example 3 shows that the assertion need not hold if $\Sigma$ is not of finite type.

Proof: Let $U \subseteq A^{\mathbb{Z}}$ be a $\mathcal{C}$-clopen set such that $\Sigma=\Omega_{F}(U)$. Let $U$ be a union of cylinders of words of length $q$. Set $\widetilde{\Omega}_{\sigma}(U)=\bigcap_{n \in \mathbb{Z}} \sigma^{n}(U)$. By a generalization of a theorem of Hurd [8] (see Kurka [15]), there exists $m>0$ such that $\Sigma=F^{m}\left(\widetilde{\Omega}_{\sigma}\right)$. If $d_{\mathcal{W}}(x, \Sigma)<1 / q$ then there exists $l>0$ such that for every $k \in \mathbb{Z}$ there exists a nonnegative $j<l$ such that $\sigma^{k+j}(x) \in U$. It follows that there exists $n>0$ such that $F^{n}(x) \in \widetilde{\Omega}_{\sigma}(U)$ and therefore $F^{n+m}(x) \in \Sigma$.

\section{Examples}

Example 1 The Identity rule $\operatorname{Id}(x)=x$.

$\left(\mathcal{B}_{A}, \operatorname{Id}_{\mathcal{B}}\right)$ and $\left(\mathcal{W}_{A}, \operatorname{Id}_{\mathcal{W}}\right)$ are chain-transitive (since both $\mathcal{B}_{A}$ and $\mathcal{W}_{A}$ are connected). However, $\left(\mathcal{C}_{A}, \mathrm{Id}\right)$ is not chain-transitive. Thus the converse of Theorem 20(6) and of Theorem 25(4) does not hold.

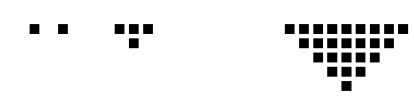

Figure 1: The product ECA184

Example 2 The product rule ECA128 $F(x)_{i}=x_{i-1} \cdot x_{i} \cdot x_{i+1}$.

$\left(\mathcal{C}_{A}, F\right),\left(\mathcal{B}_{A}, F_{\mathcal{B}}\right)$ and $\left(\mathcal{W}_{A}, F_{\mathcal{W}}\right)$ are almost equicontinous and the configuration $0^{\infty}$ is equicontinuous in all these versions. By Theorem $27,\left\{0^{\infty}\right\}$ is a $\mathcal{W}$-attractor. However, contrary to a mistaken Proposition 9 in [3], $\left\{0^{\infty}\right\}$ is not $\mathcal{B}$-attractor. For a given $0<\varepsilon<1$ define $x \in A^{\mathbb{Z}}$ by $x_{i}=1$ iff $3^{n}(1-\varepsilon)<|i| \leq 3^{n}$ for some $n \geq 0$. Then $d_{\mathcal{B}}\left(x, 0^{\infty}\right)=\varepsilon$ but $x$ is a fixed point, since $d_{\mathcal{B}}(F(x), x)=\lim _{n \rightarrow \infty} 2 n / 3^{n}=0$ (see Figure 1).

Example 3 The trafic ECA184 $F(x)_{i}=1$ iff $x_{[i-1, i]}=10$ or $x_{[i, i+1]}=11$.

No $F^{q} \sigma^{p}$ is $\mathcal{C}$-almost equicontinuous, so $\mathfrak{A}(F)=\emptyset$. However, if $d_{\mathcal{W}}\left(x, 0^{\infty}\right)<\delta$, then $d_{\mathcal{W}}\left(F^{n}(x), 0^{\infty}\right)<\delta$ for every $n>0$, since $F$ conserves the number of letters 1 in a configuration. Thus $0^{\infty}$ is a point of equicontinuity in $\left(\mathcal{T}_{A}, F_{\mathcal{T}}\right)$, 


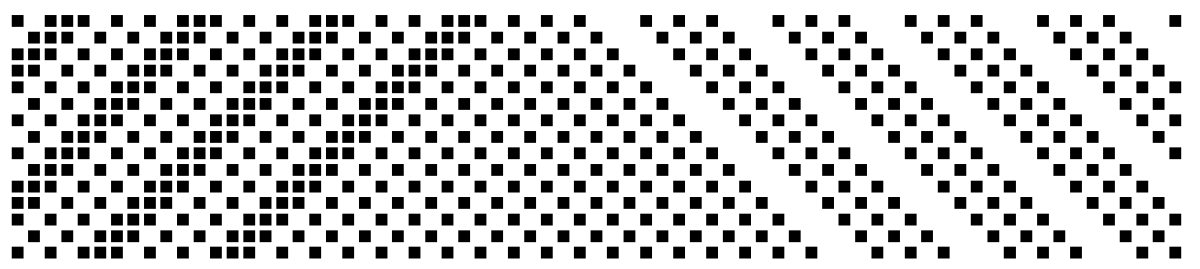

Figure 2: The traffic ECA184

$\left(\mathcal{B}_{A}, F_{\mathcal{B}}\right)$, and $\left(\mathcal{W}_{A}, F_{\mathcal{W}}\right)$. This shows that item $(2)$ of Theorems 17,20 and 25 cannot be converted. The maximal $\mathcal{C}$-attractor $\Omega_{F}=\left\{x \in A^{\mathbb{Z}}: \forall n>\right.$ $\left.0,1(10)^{n} 0 \nsubseteq x\right\}$ is not SFT. We show that it does not $\mathcal{W}$-attracts points from any of its neighbourhood. For a given even integer $q>2$ define $x \in A^{\mathbb{Z}}$ by

$$
x_{i}= \begin{cases}0 & \text { if } \exists n \geq 0, i=q n+1 \\ 1 & \text { if } \exists n<0, i=q n \\ \left((01)^{\infty}\right)_{i} & \text { otherwise }\end{cases}
$$

Then $d_{W}\left(F^{k}(x), \Omega_{F}\right)=1 / q$ for all $k>0$ (see Figure 2 , where $q=8$ ).

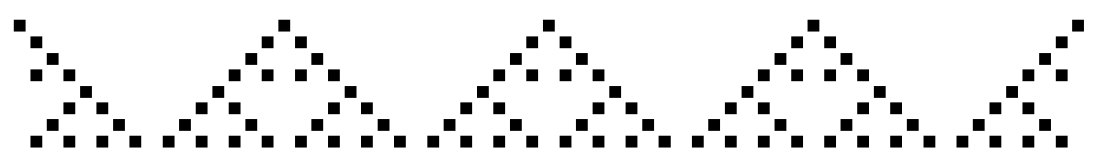

Figure 3: The sum ECA90

Example 4 The sum ECA90 $F(x)_{i}=\left(x_{i-1}+x_{i+1}\right) \bmod 2$.

Both $\left(\mathcal{B}_{A}, F_{\mathcal{B}}\right)$ and $\left(\mathcal{W}_{A}, F_{\mathcal{W}}\right)$ are sensitive (Cattaneo et al. [4]). For a given $n>0$ define a configuration $z$ by $z_{i}=1$ iff $i=k 2^{n}$ for some $k \in \mathbb{Z}$. Then $F^{2^{n-1}}(z)=(01)^{\infty}$. For any $x \in A^{\mathbb{Z}}$, we have $d_{\mathcal{W}}(x, x+z)=2^{-n}$ but $d_{\mathcal{W}}\left(F^{2^{n-1}}(x), F^{2^{n-1}}(x+z)\right)=1 / 2$. The same argument works for $\left(\mathcal{B}_{A}, F_{\mathcal{B}}\right)$.

Example 5 The shift ECA170 $F(x)_{i}=x_{i+1}$.

Since the system has fixed points $0^{\infty}$ and $1^{\infty}$, it has uncountable number of periodic points. However, the periodic points are not dense in $\mathcal{B}_{A}([2])$.

\section{Future directions}

One of the promising research directions is the connection between the generic space and the space of Borel probability measures which is based on the factor map $\Phi$. In particular Lyapunov functions based on particle weight functions (see Kưrka [13]) work both for the measure space $\mathcal{M}_{A}$ and the generic space $\mathcal{G}_{A}$. The potential of Lyapunov functions for the classification of attractors has not yet been fully explored. This holds also for the connections between attractors in different topologies. While the theory of attractors is well established in compact spaces, in noncompact spaces there are several possible approaches. Finally, the comparison of entropy properties of CA in different topologies may be revealing for classification of CA.

There is even a more general approach to different topologies for CA based on the concept of submeasure on $\mathbb{Z}$. Since each submeasure defines a pseudometric, it would be interesting to know, whether $\mathrm{CA}$ are continuous with respect to any of these pseudometrics, and whether some dynamical properties of CA can be derived from the properties of defining submeasures. 


\section{Acknowledgments}

We thank Marcus Pivato and Francois Blanchard for careful reading of the paper and many valuable suggestions. The research was partially supported by the Research Program CTS MSM 0021620845.

\section{Primary literature}

\section{References}

[1] A. S. Besicovitch. Almost periodic functions. Dover, 1954.

[2] F. Blanchard, J. Cervelle, and E. Formenti. Some results about the chaotic behaviour of cellular automata. Theoretical Computer Science, 349(3):318 336,2005 .

[3] F. Blanchard, E. Formenti, and P. Kůrka. Cellular automata in the Cantor, Besicovitch and Weyl spaces. Complex Systems, 11(2):107-123, 1999.

[4] G. Cattaneo, E. Formenti, L. Margara, and J. Mazoyer. A shift-invariant metric on $S^{\mathbb{Z}}$ inducing a nontrivial topology, volume 1295 of Lecture Notes in Computer Science. Springer-Verlag, 1997.

[5] J. Cervelle and E. Formenti. Algorithmic complexity and cellular automata. In Andrew Adamatzky, editor, Mathematical Theory of Cellular Automata, Encyclopedia of Complexity and System Sciences. SpringerVerlag, 2008.

[6] E. Formenti and P. Kůrka. Subshift attractors of cellular automata. Nonlinearity, 20:105-117, 2007.

[7] G. A. Hedlund. Endomorphisms and automorphisms of the shift dynamical system. Mathematical Systems Theory, 3:320-375, 1969.

[8] L. P. Hurd. Recursive cellular automata invariant sets. Complex Systems, 4:119-129, 1990.

[9] A. Iwanik. Weyl almost periodic points in topological dynamics. Colloquium Mathematicum, 56:107-119, 1988.

[10] T. Kamae. Normal numbers and ergodic theory. In Proceedings of the Third Japan-USSR Symposium on Probability Theory, (Tashkent, 1975), volume 550 of Lecture Notes in Mathematics, pages 253-269. SpringerVerlag, Berlin, 1976.

[11] C. Knudsen. Chaos without nonperiodicity. American Mathematical Monthly, 101:563-565, 1994.

[12] P. Kůrka. Languages, equicontinuity and attractors in cellular automata. Ergodic Theory and Dynamical Systems, 17:417-433, 1997.

[13] P. Kůrka. Cellular automata with vanishing particles. Fundamenta Informaticae, 58:1-19, 2003.

[14] P. Kůrka. On the measure attractor of a cellular automaton. Discrete and Continuous Dynamical Systems, supplement volume 2005:524-535, 2005.

[15] P. Kůrka. Topological dynamics of one-dimensional cellular automata. In Andrew Adamatzky, editor, Mathematical Theory of Cellular Automata, Encyclopedia of Complexity and System Sciences. Springer-Verlag, 2008. 
[16] J. Marcinkiewicz. Une remarque sur les espaces de a.s. Besicovitch. C. R. Acad. Sc. Paris, 208:157-159, 1939.

[17] M. Pivato. The ergodic theory of cellular automata. In Andrew Adamatzky, editor, Mathematical Theory of Cellular Automata, Encyclopedia of Complexity and System Sciences. Springer-Verlag, 2008.

[18] M. Sablik. Étude de l'action conjointe d'un automate cellulaire et du décalage: une approche topologique et ergodique. $\mathrm{PhD}$ thesis, Université de la Mediterranée, Marseille, 2006.

\section{Books and reviews}

\section{References}

[1] A. S. Besicovitch. Almost periodic functions. Dover, 1954.

[2] B. P. Kitchens. Symbolic Dynamics. Springer-Verlag, Berlin, 1998.

[3] P. Kůrka. Topological and symbolic dynamics, volume 11 of Cours spécialisés. Société Mathématique de France, Paris, 2003.

[4] D. Lind and B. Marcus. An introduction to symbolic dynamics and coding. Cambridge University Press, Cambridge, 1995. 